\title{
The Association Between Insulin Initiation and Adverse Outcomes After Hospital Discharge in Older Adults: a Population-Based Cohort Study
}

\author{
Zoe Lysy, MD, MPH', ${ }^{1,}$ Kinwah Fung, MSc ${ }^{3}$, Vasily Giannakeas, MSc ${ }^{1,3}$, Hadas D. Fischer, MD, MSc ${ }^{3}$, \\ Chaim M. Bell, MD, PhD $2,3,4,5$, and Lorraine L. Lipscombe, MD, MSc 1,2,3,4
}

'Women's College Hospital, Women's College Research Institute, Toronto, ON, Canada; '2Department of Medicine, University of Toronto, Toronto, Canada; ${ }^{3}$ Institute for Clinical Evaluative Sciences, Toronto, Canada; ${ }^{4}$ Institute of Health Policy, Management and Evaluation, University of Toronto, Toronto, Canada; ${ }^{5}$ Sinai Health System, Toronto, Canada.

\begin{abstract}
BACKGROUND: Starting insulin therapy in hospitalized patients may be associated with an increase in serious adverse events after discharge.

OBJECTIVE: Determine whether post-discharge risks of death and rehospitalization are higher for older hospitalized patients prescribed new insulin therapy compared with oral hypoglycemic agents (OHAs).

DESIGN: Retrospective population-based cohort study including hospital admissions in Ontario, Canada, between April 1, 2004, and Nov 30, 2013.

PATIENTS: Persons aged 66 and over discharged after a hospitalization and dispensed a prescription for insulin and/or an OHA within 7 days of discharge. We included 104,525 individuals, subcategorized into four mutually exclusive exposure groups based on anti-hyperglycemic drug use in the 7 days post-discharge and the 365 days prior to the index admission.
\end{abstract}

MAIN MEASURES: Prescriptions at discharge were categorized as new insulin (no insulin before admission), prevalent insulin (prescribed insulin before admission), new OHA(s) (no OHA or insulin before admission), and prevalent OHA (prescribed OHA only before admission) as the referent category. The primary and secondary outcomes were 30-day deaths and emergency department (ED) visits or readmissions respectively.

KEY RESULTS: Of 104,525 patients, 9.2\% were initiated on insulin, $4.1 \%$ died, and $26.2 \%$ had an ED visit or readmission within 30 days of discharge. Deaths occurred in $7.14 \%$ of new insulin users, $4.86 \%$ of prevalent insulin users, $3.25 \%$ of new OHA users, and $3.45 \%$ of prevalent OHA users. After adjustment for covariates, new insulin users had a significantly higher risk of death (adjusted hazard ratio (aHR) 1.59, 95\% confidence interval (CI) 1.46 to 1.74 ) and ED visit/readmissions (aHR 1.17, 95\% CI 1.12 to 1.22 ) than prevalent OHA users.

CONCLUSIONS: Initiation of insulin therapy in older hospitalized patients is associated with a higher risk of death and ED visits/readmissions after discharge, highlighting a need for better transitional care of insulin-treated patients.

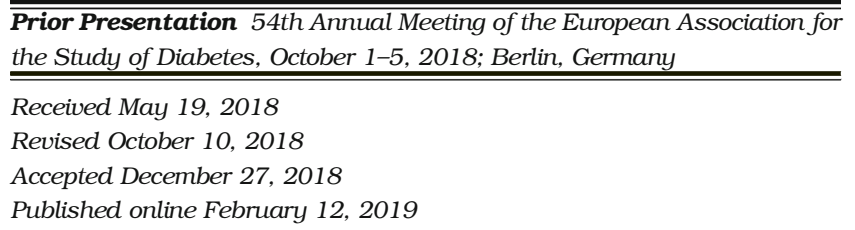

the Study of Diabetes, October 1-5, 2018; Berlin, Germany

Published online February 12, 2019
KEY WORDS: diabetes; care transitions; health services research.

J Gen Intern Med 34(4):575-82

DOI: $10.1007 /$ s11606-019-04849-3

(c) Society of General Internal Medicine 2019

\section{BACKGROUND}

Starting insulin therapy in older persons can be dangerous if ongoing support is not ensured. The Institute for Safe Medication Practices lists insulin as a "high-alert" medication, whereby it can cause significant harm if used in error. ${ }^{1}$ Insulin is often initiated in hospitalized patients to manage hyperglycemia $^{2}$, which can be continued at discharge to improve longterm glycemic control. Previous clinical trials have shown that intensive glycemic control reduces long-term risk of microvascular complications. ${ }^{3,4}$ Moreover, insulin treatment of hospitalized cardiac patients decreases mortality after discharge ${ }^{5,6}$ although this has not always been confirmed. ${ }^{7}$ These benefits may be offset by a higher risk of hypoglycemia and associated adverse events with very low glycemic targets ${ }^{8}$ especially in heterogeneous patient populations. ${ }^{9,}{ }^{10}$ Observational studies show that insulin therapy is associated with a higher risk of hypoglycemia, cardiovascular events, and death compared with other glucose-lowering agents. ${ }^{11-16}$ Hypoglycemia events now exceed hyperglycemic presentations to acute care $^{17}$ particularly in elderly, comorbid patients. ${ }^{9}, 18$ Substantial education and support are required for patients and their families initiated on insulin in hospital, to ensure safe use of insulin after discharge. ${ }^{19}$ This instruction is often given at a time of competing interests during an inpatient admission, and insulin needs may change after discharge as patients return to their usual lifestyle and health status. Ensuring ongoing insulin support is thus essential during the discharge transition to maintain adequate glycemia and reduce adverse events. Evidence regarding outcomes after discharge of insulin-treated patients is conflicting and little is known about adverse events in patients newly prescribed insulin at hospital discharge.

This study evaluated the risk of adverse outcomes after a hospitalization among older patients who were discharged on 
anti-hyperglycemic therapy. Our objective was to determine whether the 30-day post-discharge risk of death and emergency department (ED) visits or readmissions were higher in patients newly started on insulin compared with patients taking oral hypoglycemic agents (OHAs). To determine the specificity of the association between new insulin use and adverse outcomes, we also evaluated outcomes in new OHA users and in patients who were already taking insulin at admission.

\section{METHODS AND STATISTICAL ANALYSIS}

We conducted a population-based, retrospective cohort study using administrative health care databases from the province of Ontario, Canada. These databases include over 1,878,325 residents aged 66 years and older, for whom most prescription medications are captured through the provincial drug plan.

\section{Data Sources}

Hospital admissions were identified using the Canadian Institute for Health Information Discharge Abstract Database (CIHI-DAD), which contains data on up to 25 International Classification of Disease (ICD)-10 diagnosis codes, and the National Ambulatory Care Reporting System (NACRS) to identify emergency department (ED) visits. Prescriptions were captured using the Ontario Drug Benefit (ODB) database, which contains records of all outpatient prescriptions for individuals aged 65 and older. ${ }^{20}$ We used the Ontario Health Insurance Plan (OHIP) database to identify physician claims and the Registered Persons Database (RPDB) to obtain demographic information and deaths. These datasets were linked using personal unique encoded identifiers and analyzed at the Institute for Clinical Evaluative Sciences (ICES).

\section{Study Population and Cohort Definition}

The study population included patients aged 66 years and older discharged alive from a non-elective hospitalization episode between April 1, 2004, and November 30, 2013, and dispensed a prescription of insulin or an OHA within 7 days of their discharge date. The age group $66+$ provides a 1-year look-back for database coverage as of age 65 years. When multiple admissions fit the criteria for a given patient, a random hospital episode was chosen using a random number generator. We excluded patients admitted to/from another facility (e.g., rehabilitation, continuous complex care, mental health institution, palliative care) due to differing goals of care and hospitalizations that were greater than 30 days because of their more complex discharge issues.

\section{Drug Exposure}

Anti-hyperglycemic drug exposure was categorized into four mutually exclusive groups, according to prescriptions dispensed in the 7 days post-discharge and in the 365 days before the index hospitalization: (1) new insulin use, $\geq 1$ insulin prescription ( \pm OHA) post-discharge and no insulin ( \pm OHA) before hospitalization; (2) prevalent insulin use, $\geq 1$ insulin prescription ( \pm OHA) post-discharge and before hospitalization; (3) new OHA use, $\geq 1$ prescription for $\geq 1$ OHA only postdischarge and no anti-hyperglycemic medication before hospitalization; and (4) prevalent OHA use, $\geq 1$ prescription for $\geq 1$ OHA only post-discharge and before hospitalization. The latter category served as the referent to which all other exposures were compared, due to their lower risk for adverse events. Patients who had been dispensed insulin before admission but not after discharge were excluded, as we could not determine which of those patients had insulin discontinued in hospital versus those in whom no changes were made.

\section{Study Outcomes}

The primary outcome was all-cause mortality within 30 days of the index date (discharge). Deaths were identified using the RPDB enriched with the hospital discharge abstract database for deaths that occurred in a subsequent hospitalization. As secondary outcomes, we examined 30-day ED visits or readmissions for any cause and for hypo/hyperglycemia (ICD-10 code R739, E10-E16) as the main diagnosis. The composite outcome of 30-day deaths and ED visits/readmissions was also recorded.

\section{Covariates}

We identified sex, age, low-income (determined by linking neighborhood postal code) and long-term care residence flagged by the ODB database, rural residence, Charlson Comorbidity Score, ${ }^{21}$ number of unique medications dispensed in the prior year, ${ }^{22}$ dementia, cardiovascular disease and chronic renal failure in the prior 5 years, index admission to a teaching hospital, consultation with an endocrinologist during index admission, and length of stay. LACE+ index was determined for each individual (a validated score to predict post-discharge death or readmission $\left.{ }^{23,}{ }^{24}\right)$ : this incorporates length of hospital stay (L), acuity of admission (A), comorbidity $(\mathrm{C})$, and emergency department utilization in the prior 6 months (E), patient age and sex, hospital teaching status, acute diagnoses and procedures, number of alternative level of care days, and number of admissions in the prior year.

\section{Statistical Analysis}

The cumulative incidence of each outcome by the exposure group was calculated using Kaplan-Meier survival analyses. We performed Cox proportional hazards regression analyses to compare primary and secondary outcomes in new insulin users, new OHA users, and prevalent users to the referent group (prevalent OHA use). Cause-specific hazard ratios (HRs) were estimated to account for competing risk of death when analyzing ED visits/readmissions. Crude HRs were calculated and adjusted for all covariates except for Charlson Comorbidity Score, hospital length of stay, and unique number of medications due to their potential high correlation with the LACE+ index. ${ }^{23,}{ }^{24}$ We tested for an interaction between an endocrinologist consultation and drug exposure to 


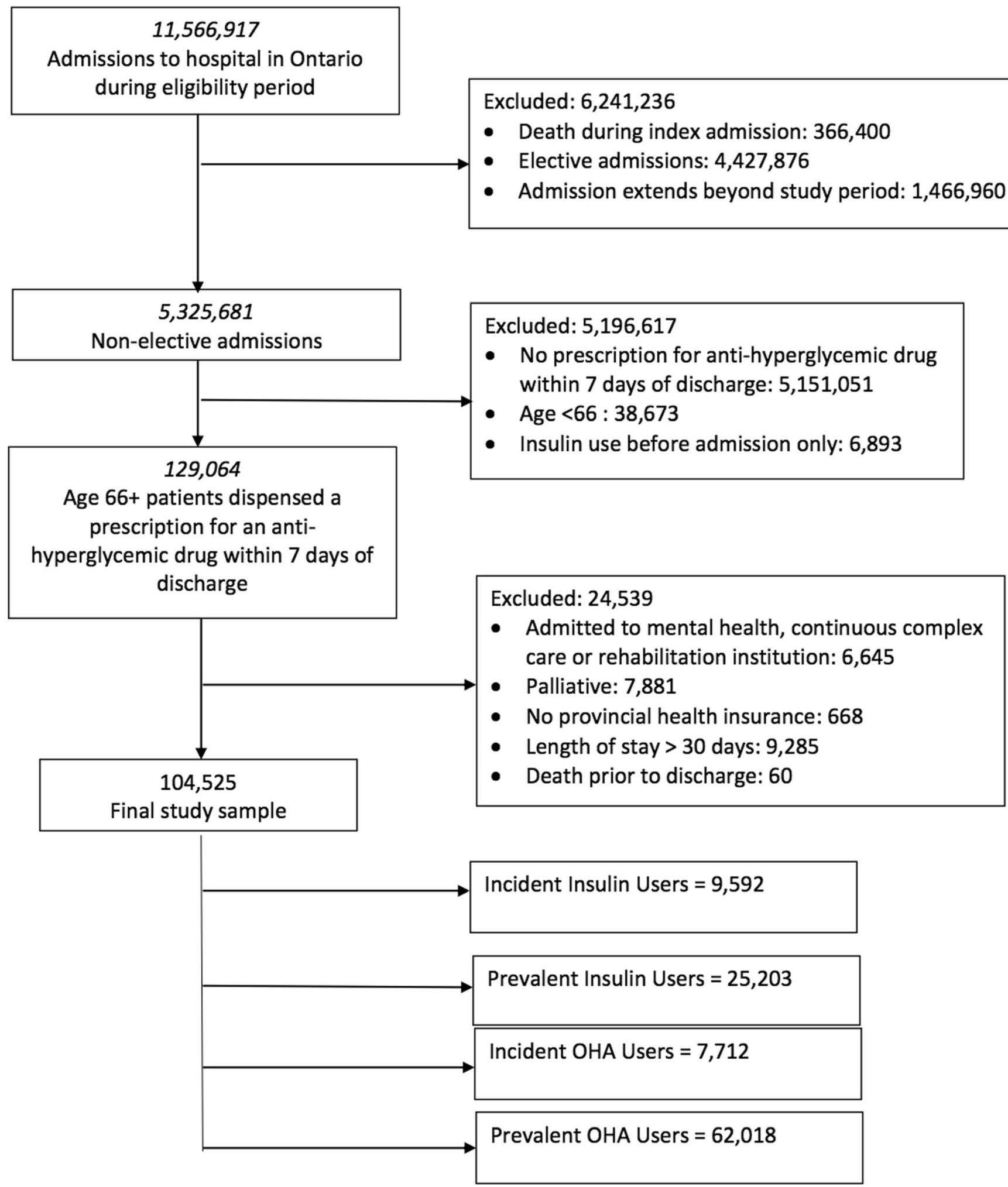

Figure 1 Participant flow diagram of cohort creation and exclusions.

determine the differential effect of specialized inpatient diabetes support on outcomes, and performed stratified analyses for significant interactions.

Sensitivity Analyses. There were three sensitivity analyses conducted as part of this study. First, we repeated analyses stratifying index hospitalizations by most responsible diagnosis: (1) cardiovascular disease (ICD10 code I20-25), (2) diabetes (ICD-10 code E10-E14), and (3) other. Second, to address the possibility of differential care in more frail patients, we repeated all analyses excluding patients with dementia or long-term 
care residence. Third, we repeated all analyses using a 90-day window to determine robustness of effects over a longer period. All analyses were conducted using SAS version 9.4 (SAS Institute Inc., Cary, NC). All statistical tests were two-sided with a significance level of 0.05 to denote statistical significance.

\section{RESULTS}

\section{Baseline Data}

Our study population included 104,525 individuals discharged from hospital between April 1, 2004, and November 30, 2013, who fulfilled the inclusion criteria (Fig. 1). Of these, 9592 $(9.2 \%)$ were new insulin users, $25,203(24.1 \%)$ were prevalent insulin users, 7712 (7.4\%) were new OHA users, and 62,018 $(59.3 \%)$ were prevalent OHA users (Table 1). Prevalent insulin users had the highest LACE+ and Charlson Comorbidity Score, had the highest rates of cardiovascular disease and chronic renal failure, and were on the highest number of medications. In contrast, dementia and LTC residence were most common in new insulin users. New insulin users also had a longer median length of stay than other groups.

\section{Adverse Outcomes After Discharge}

There were 4297 deaths (4.1\%) within 30 days after discharge. Of the deaths, $50.6 \%$ occurred out of hospital; $49.5 \%$ of hospital visits resulted in a readmission. New insulin users had the highest 30 -day mortality $(7.1 \%, 95 \%$ confidence interval (CI) 6.64-7.67) and proportion of out-of-hospital deaths (60.1\%), followed by prevalent insulin users and new OHA users.
There were 27,382 (26.2\%) ED visits or readmissions; 767 (2.8\%) were for hypo/hyperglycemia. ED visit/readmission rates were also higher among new $(27.6 \%, 95 \%$ CI $27.2-$ $29.0)$ and prevalent $(29.5 \%, 95 \%$ CI $29.2-30.3)$ insulin users than among new $(25.0 \%, 95 \%$ CI 24.2-26.1) and prevalent (24.8\%, 95\% CI 24.6-25.3) OHA users (Table 2).

Primary Analysis. After adjusting for covariates, 30-day mortality was significantly higher for all exposure groups compared with prevalent OHA users (Table 2). New insulin use was associated with the highest risk of death (adjusted HR (aHR) 1.59, 95\% CI 1.46-1.74); prevalent insulin use and new OHA use were associated with adjusted HRs for 30-day mortality of 1.12 (95\% CI $1.04-1.21)$ and 1.26 (95\% CI $1.11-1.44)$ respectively.

The risk of ED visits or readmissions was also significantly higher in all exposure groups compared with prevalent $\mathrm{OHA}$ users (new insulin: aHR 1.17, 95\% CI 1.12-1.22; prevalent insulin: aHR 1.15, 95\% 1.11-1.18; and new OHA: aHR 1.05, 95\% CI 1.00-1.10). Similar trends were seen for the composite outcome. When only ED visits/readmissions for hypo/ hyperglycemia were considered, the risk for new insulin users was over twofold higher than that for prevalent OHA users (Table 2). We did not find collinearity between the LACE+ score and other variables on adjusted analyses. There was a significant interaction between an endocrinologist consultation and drug exposure on mortality; however, this was no longer significant once adjusted for other covariates.

Sensitivity Analyses. Results were similar when we restricted analyses to index admissions for cardiac and other causes (Fig. 2), although among cardiac admissions only new

Table 1 Baseline Characteristics, by Medication Exposure Group

\begin{tabular}{|c|c|c|c|c|}
\hline & $\begin{array}{l}\text { Prevalent OHA } \\
N=62,108\end{array}$ & $\begin{array}{l}\text { New insulin } \\
N=9592\end{array}$ & $\begin{array}{l}\text { Prevalent insulin } \\
N=25,203\end{array}$ & $\begin{array}{l}\text { New OHA } \\
N=7712\end{array}$ \\
\hline \multicolumn{5}{|l|}{ Demographic variables } \\
\hline Age (years), mean (SD) & $79.1(7.3)$ & $78.4(7.6)$ & $77.6(7.0)$ & $77.5(7.4)$ \\
\hline Female sex, $N(\%)$ & $31,313(50.5 \%)$ & $4733(49.3 \%)$ & $13,127(52.1 \%)$ & $3531(45.8 \%)$ \\
\hline Rural residence, $N(\%)$ & $9316(15.0 \%)$ & $1133(11.8 \%)$ & $3844(15.3 \%)$ & $1240(16.1 \%)$ \\
\hline Low-income status, $N(\%)$ & $21,479(34.6 \%)$ & $2910(30.3 \%)$ & $8193(32.5 \%)$ & $1889(24.5 \%)$ \\
\hline Long-term care residence, $N(\%)$ & $6819(11.0 \%)$ & $2199(22.9 \%)$ & $4936(19.6 \%)$ & $401(5.2 \%)$ \\
\hline \multicolumn{5}{|l|}{ Comorbidities } \\
\hline Charlson Score $^{\mathrm{a}}$, mean (SD) & $2.29(1.51)$ & $2.66(1.66)$ & $2.74(1.60)$ & $2.23(1.49)$ \\
\hline LACE plus index ${ }^{\mathrm{b}}$, mean (SD) & $67.8(14.8)$ & $68.5(15.1)$ & $71.7(14.8)$ & $63.8(15.1)$ \\
\hline Dementia, $N(\%)$ & $15,820(25.5 \%)$ & $3007(31.3 \%)$ & $7290(28.9 \%)$ & $1181(15.3 \%)$ \\
\hline Cardiovascular disease, $N(\%)$ & $31,182(50.3 \%)$ & $4370(45.6 \%)$ & $15,086(59.9 \%)$ & $3592(46.6 \%)$ \\
\hline Chronic renal failure, $N(\%)$ & $16,216(26.1 \%)$ & $3813(39.8 \%)$ & $12,662(50.2 \%)$ & $1337(17.3 \%)$ \\
\hline Number unique medications, median (IQR) & $13(9-17)$ & $12(9-17)$ & $16(12-21)$ & $8(4-14)$ \\
\hline \multicolumn{5}{|l|}{ Index hospitalization } \\
\hline \multicolumn{5}{|l|}{ Main diagnosis } \\
\hline Cardiac disease, $N(\%)$ & $9637(15.5 \%)$ & $1280(13.3 \%)$ & $4697(18.6 \%)$ & $1278(16.6 \%)$ \\
\hline Diabetes, $N(\%)$ & $2541(4.1 \%)$ & $2204(23.0 \%)$ & $2730(10.8 \%)$ & $929(12.0 \%)$ \\
\hline Other, $N(\%)$ & $49,840(80.4 \%)$ & $6108(63.7 \%)$ & $17,776(70.5 \%)$ & $5505(71.4 \%)$ \\
\hline Length of hospital stay (days), median (IQR) & $6(3-10)$ & $9(5-15)$ & $7(4-12)$ & $7(4-12)$ \\
\hline Teaching hospital, $N(\%)$ & $15,644(25.2 \%)$ & $2265(23.6 \%)$ & $6310(25.0 \%)$ & $1963(25.5 \%)$ \\
\hline Endocrinologist consultation, $N(\%)$ & $5274(8.5 \%)$ & $2637(27.5 \%)$ & $3638(14.4 \%)$ & $1246(16.2 \%)$ \\
\hline
\end{tabular}

$N$ number, OHA oral hypoglycemic agents

${ }^{a}$ The Charlson Comorbidity Index is a method of weighting comorbidities based on ICD diagnostic codes, to derive a comorbidity score for a patient

${ }^{b}$ LACE plus index is a score to predict risk of post-discharge death or non-elective readmission using health care administrative data validated in the Ontario population 
Table 2 Mortality and Hospital Visit Outcomes at 30 Days by Medication Exposure Group, Univariate and Multivariable Cox Proportional Hazard Models, Complete Cohort $(N=104,525)$

\begin{tabular}{|c|c|c|c|c|}
\hline Outcomes & $\begin{array}{l}\text { Prevalent OHA (referent) } \\
N=62,018\end{array}$ & $\begin{array}{l}\text { New insulin } \\
N=9592\end{array}$ & $\begin{array}{l}\text { Prevalent insulin } \\
N=\mathbf{2 5 , 2 0 3}\end{array}$ & $\begin{array}{l}\text { New OHA } \\
N=7712\end{array}$ \\
\hline \multicolumn{5}{|l|}{ All-cause mortality, 30 days } \\
\hline Events, $N(\%)^{\mathrm{a}}$ & $2137(3.45 \%)$ & $685(7.14 \%)$ & $1224(4.86 \%)$ & $251(3.25 \%)$ \\
\hline Out-of-hospital deaths, $N(\%)$ & $1026(48.0 \%)$ & $412(60.1 \%)$ & $638(52.1 \%)$ & $100(39.8 \%)$ \\
\hline In-hospital deaths, $N(\%)$ & $1111(52.0 \%)$ & $273(39.9 \%)$ & $586(47.9 \%)$ & $151(60.2 \%)$ \\
\hline Unadjusted HR (95\% CI) & 1.00 & $2.12(1.94-2.31)$ & $1.42(1.32-1.52)$ & $0.94(0.83-1.08)$ \\
\hline Adjusted HR $(95 \% \mathrm{CI})^{\mathrm{b}}$ & 1.00 & $1.59(1.46-1.74)$ & $1.12(1.04-1.21)$ & $1.26(1.11-1.44)$ \\
\hline$p$ value, adjusted HR & - & $<0.0001$ & 0.0022 & 0.0005 \\
\hline \multicolumn{5}{|c|}{ Any ED visit or readmission, 30 days } \\
\hline Events, $N(\%)^{\mathrm{a}}$ & $15,386(25.0 \%)$ & $2644(28.1 \%)$ & $7425(29.8 \%)$ & $1927(25.1 \%)$ \\
\hline ED visits only, $N(\%)$ & $7828(50.9 \%)$ & $1322(50.0 \%)$ & $3636(49.0 \%)$ & $1049(54.4 \%)$ \\
\hline Readmissions, $N(\%)$ & $7558(49.1 \%)$ & $1322(50.0 \%)$ & $3789(51.1 \%)$ & $878(45.6 \%)$ \\
\hline Unadjusted HR (95\% CI) & 1.00 & $1.14(1.10-1.19)$ & $1.22(1.19-1.25)$ & $1.00(0.96-1.05)$ \\
\hline Adjusted HR $(95 \% \mathrm{CI})^{\mathrm{b}}$ & 1.00 & $1.17(1.12-1.22)$ & $1.15(1.11-1.18)$ & $1.05(1.00-1.10)$ \\
\hline$p$ value, adjusted $\mathrm{HR}$ & - & $<0.0001$ & $<0.0001$ & 0.0529 \\
\hline \multicolumn{5}{|c|}{ ED visit or admission for hypo/hyperglycemia, 30 days } \\
\hline Events, $N(\%)^{\mathrm{a}}$ & $302(0.49 \%)$ & $113(1.21 \%)$ & $289(1.17 \%)$ & $63(0.82 \%)$ \\
\hline Unadjusted HR (95\% CI) & 1.00 & $2.47(1.99-3.06)$ & $2.37(2.02-2.79)$ & $1.68(1.28-2.20)$ \\
\hline Adjusted HR $(95 \% \mathrm{CI})^{\mathrm{b}}$ & 1.00 & $2.49(2-3.1)$ & $2.32(1.96-2.75)$ & $1.72(1.31-2.26)$ \\
\hline$p$ value, adjusted HR & - & $<0.0001$ & $<0.0001$ & $<0.0001$ \\
\hline \multicolumn{5}{|c|}{ Composite outcome (death or ED visit/readmission), 30 days } \\
\hline Events, $N(\%)^{\mathrm{a}}$ & $16,195(26.1 \%)$ & $2975(31.0 \%)$ & $7932(31.5 \%)$ & $2009(26.1 \%)$ \\
\hline Unadjusted HR (95\% CI) & 1.00 & $1.22(1.17-1.27)$ & $1.24(1.21-1.27)$ & $0.99(0.95-1.04)$ \\
\hline Adjusted HR $(95 \% \mathrm{CI})^{\mathrm{b}}$ & 1.00 & $1.21(1.16-1.26)$ & $1.15(1.12-1.18)$ & $1.06(1.01-1.11)$ \\
\hline$p$ value, adjusted HR & - & $<0.0001$ & $<0.0001$ & 0.0168 \\
\hline
\end{tabular}

$N$ number, OHA oral hypoglycemic agents, HR hazard ratio, CI confidence intervals, ED emergency department

${ }^{a}$ Cumulative incidence of events over 30-day time period

${ }^{b}$ Model adjusted for sex, age, low-income status, rural residence, long-term care, cardiovascular disease, chronic renal failure, dementia, LACE+ score, and teaching hospital at discharge

insulin users had a significantly increased mortality (Fig. 2a). Conversely, when we restricted to diabetes admissions, the association between new insulin and post-discharge outcomes largely disappeared. Findings were similar when we excluded patients with dementia or LTC residence, and when the postdischarge window was extended to 90 days.

\section{CONCLUSIONS}

We found a significantly higher 30-day mortality and ED visit or readmission risk in older patients who were discharged from

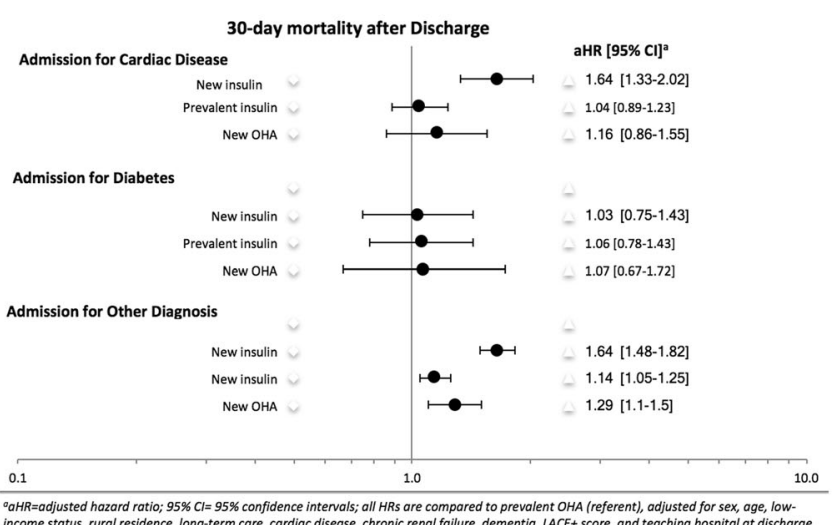

hospital on insulin therapy. The highest risk was seen among patients newly started on insulin, who had a mortality rate of $7.1 \%$ and $28 \%$ probability of presenting to hospital within 30 days. Even after adjustment for important confounders, the risk of death was an estimated 1.5 times higher for new insulin users compared with patients taking OHAs. These findings are consistent with previous observational studies of greater mortality with insulin therapy. ${ }^{11-13,15,16}$ Prior studies were largely in outpatient settings and did not isolate effects of insulin initiation, ${ }^{11-13,}{ }^{16}$ making it difficult to separate confounding by indication. Our study was unique in isolating new from prevalent insulin users, who had a higher mortality despite

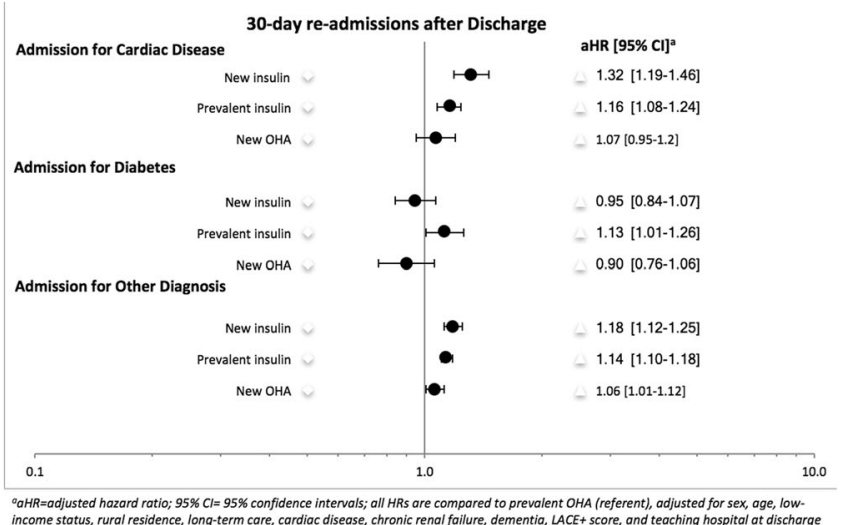

Figure 2 a Forest plot of adjusted hazard ratios for 30-day mortality after discharge for each medication exposure group, stratified by reason for index hospital admission. b Forest plot of adjusted hazard ratios for 30-day ED visits or readmissions after discharge for each medication exposure group, stratified by reason for index hospital admission. ${ }^{a}$ aHR, adjusted hazard ratio; $95 \%$ CI, $95 \%$ confidence intervals. All HRs are compared with prevalent OHA (referent), adjusted for sex, age, low-income status, rural residence, long-term care, cardiac disease, chronic renal failure, dementia, LACE+ score, and teaching hospital at discharge. 
lower comorbidity than prevalent insulin users. Our findings provide new evidence regarding risks associated with new insulin initiation in older patients discharged from hospital.

The association between insulin therapy and higher mortality has been attributed to an increased risk of cardiovascular events related to hypoglycemia. ${ }^{9,10,25}$ Our study found that patients initiated on insulin after a cardiac admission had the highest mortality after discharge. This study adds to existing evidence that the benefits of interventions seen in clinical trials may be outweighed by greater harms, when applied in realworld settings with more variable patient populations. ${ }^{26}$

Interestingly, we did not see a higher risk of adverse events in insulin-treated patients who were hospitalized with diabetes as their main diagnosis. It is possible that patients received greater education, post-discharge support, and tailored treatment when diabetes was the main focus of admission. This did not affect readmissions in our study though inpatient diabetes education has been associated with reduced readmissions ${ }^{27,} 28$ particularly in incident insulin users, ${ }^{27}$ suggesting that adverse outcomes after insulin initiation may be preventable with more enhanced diabetes care during the hospitalization.

Our study should not signal a need to avoid insulin in hospitalized patients, as there are clear benefits of insulin therapy in settings of close follow-up ${ }^{5,6}$ : an admission is an opportune time for intensification of glycemic control. ${ }^{29,}{ }^{30}$ Our findings show that the good intentions to optimize diabetes in hospital may be derailed after discharge without adequate transition care. Patients may be discharged on insulin with limited self-management skills, in metabolic flux, or with multiple medication changes. There is good evidence that structured discharge planning reduces length of stay and readmissions. ${ }^{31-33}$

Older patients with diabetes being discharged from hospital often report difficulty managing medications and greater coping difficulty associated with a higher risk of unplanned health encounters. ${ }^{34}$ Discharge planning may be inadequate for complex diabetes patients, and insulin initiation especially requires effective education and follow-up due to the high risk of adverse events. Our findings raise the question of what constitutes ideal transition care or post-discharge follow-up in patients with diabetes in whom medications such as insulin are started in hospital. The utility of post-discharge clinics, discharge planning, or close health care team follow-up has well been demonstrated in chronic conditions such as heart failure and general medicine admissions. ${ }^{35-37}$ The evidence supporting such transition care in diabetes is limited but small studies do suggest benefit in terms of readmission, particularly in patients for whom the reason for admission was diabetes. ${ }^{27,} 38,39$ Qualitative studies in this population suggest that discharge instructions and ongoing support decrease readmissions in patients with diabetes. ${ }^{38,40}$ Our study was not able to accurately capture post-discharge follow-up; therefore, further research is needed to determine the nature and intensity of transition care needed for insulin-treated patients after hospital discharge.

While intensive glycemic control has great benefits, strict targets and insulin initiation may not always be appropriate, particularly in older patients with comorbidity or limited life expectancy ${ }^{19}$ or in whom proper discharge planning cannot be assured. The majority of older diabetes patients with complex health status continue to be intensively managed, despite the harms outweighing the benefits. ${ }^{41,} 42$ A US report estimated that 97,648 hospital visits are for insulin-induced hypoglycemia every year, with one-third resulting in hospitalization and $60 \%$ causing neurologic sequelae. ${ }^{18}$ The highest risks were seen in elderly insulin-treated patients. ${ }^{18}$ The benefits of initiating insulin therapy in older, comorbid, or critically ill patients therefore need to be carefully weighed with individual risks. $^{43}$

This study did have some limitations. First, we could not be certain that the new prescriptions filled at discharge were initiated in hospital. This was addressed by defining drug exposure within the first 7 days of discharge, thereby closely associating the prescription with hospitalization. ${ }^{44}$ Patients who did not fill an insulin prescription after admission may represent a higher-risk group whose insulin was stopped; however, we did not find differences in their baseline variables or outcomes. Second, we did not have information on potential confounders (glycemic control, illness severity, or patient preference). Our finding of higher risk with new versus prevalent insulin users reduces this concern, as the latter tended to have a greater comorbidity burden. Third, we could not determine whether prevalent insulin or OHA users had had a major change in their regimen during their hospitalization, or whether patients who filled their prescriptions actually took their medication. Fourth, we did not have data on post-discharge follow-up patterns. We do not believe that these limitations invalidate our main conclusions given the large sample size and robustness of the findings across sensitivity analyses.

Gaps in care continuity are an important area for patient safety. We found that one in 12 patients discharged from hospital that filled a prescription for insulin died and more than one-third had an ED visit or readmission. This effect was higher for patients started on insulin as compared with those on oral hypoglycemic agents. While we could not determine causality between insulin and mortality, we highlight a vulnerable population which needs additional resources in the discharge transition period. Further inquiry should determine appropriate interventions to reduce adverse outcomes after insulin initiation in older hospitalized patients, so that the benefits of effective diabetes management while in hospital are maintained when patients leave the hospital.

\section{Acknowledgements:}

We thank IMS Brogan Inc. for the use of their drug information database.

Contributors: $L L$ and $Z L$ had the original idea for the study and contributed to the development of the data. HF, LL, ZL, and CB designed the study. KF and VG extracted data from the source database and validated the diagnostic codes from the database. $Z L, K F$, and VG undertook the statistical analysis. ZL reviewed the literature and wrote the first draft of the manuscript. LL co-drafted the manuscript and provided oversight for the project. $C B, H F$, and $L L$ provided 
critical input to the analysis and design. All authors contributed to the critical review of the manuscript and approved its final submission. ZL and $L L$ act as guarantors for the study.

Corresponding Author: Lorraine L. Lipscombe, MD, MSc; Women's College Hospital, Women's College Research Institute, 76 Grenville St, Toronto, ON M5S 1B2, Canada (e-mail: Lorraine. Lipscombe@wchospital.ca).

Funding Dr. Lipscombe is supported by a Diabetes Canada Investigator Award. This study was conducted with the support from a CIHR operating grant (MOP No. 123263). This study was supported by ICES, which is funded by an annual grant from the Ontario Ministry of Health and Long-Term Care (MOHLTC). The opinions, results and conclusions reported in this paper are those of the authors and are independent from the funding sources. No endorsement by ICES or the Ontario MOHLTC is intended or should be inferred.

\section{Compliance with Ethical Standards:}

Conflict of Interest: The authors declare that they do not have a conflict of interest.

Ethics Approval: This project was approved by the Institutional Review Board at Sunnybrook Hospital.

Disclaimer: Parts of this material are based on data and/or information compiled and provided by the Canadian Institute for Health Information (CIHI); however, the analyses, conclusions, opinions, and statements expressed in the material are those of the authors, and not necessarily those of CIHI.

Publisher's Note: Springer Nature remains neutral with regard to jurisdictional claims in published maps and institutional affiliations.

Prior Presentation: 54th Annual Meeting of the European Association for the Study of Diabetes, October 1-5, 2018; Berlin, Germany

\section{REFERENCES}

1. Practices IfSM. ISMP List of High-Alert Medications in Acute Care Settings; https://www.ismp.org/sites/default/files/attachments/201810/highAlert2018new-Oct2018-v1.pdf Accessed Nov 24, 2018

2. Inzucchi SE. Clinical practice. Management of hyperglycemia in the hospital setting. N Engl J Med 2006;355(18):1903-1911.

3. Gerstein HC, Pogue J, Mann JF, et al. The relationship between dysglycaemia and cardiovascular and renal risk in diabetic and nondiabetic participants in the HOPE study: a prospective epidemiological analysis. Diabetologia 2005;48(9): 1749-1755.

4. UK Prospective Diabetes Study (UKPDS) Group. Intensive blood-glucose control with sulphonylureas or insulin compared with conventional treatment and risk of complications in patients with type 2 diabetes (UKPDS 33). Lancet. 1998;352(9131):837-853.

5. Ritsinger V, Malmberg $\mathbf{K}$, Martensson A, Ryden L, Wedel H, Norhammar A. Intensified insulin-based glycaemic control after myocardial infarction: mortality during 20 year follow-up of the randomised Diabetes Mellitus Insulin Glucose Infusion in Acute Myocardial Infarction (DIGAMI 1) trial. Lancet Diabetes Endocrinol 2014;2(8):627-633.

6. Malmberg K. Prospective randomised study of intensive insulin treatment on long term survival after acute myocardial infarction in patients with diabetes mellitus. DIGAMI (Diabetes Mellitus, Insulin Glucose Infusion in Acute Myocardial Infarction) Study Group. BMJ 1997;314(7093):1512-1515.

7. Malmberg $\mathbf{K}$, Ryden $\mathbf{L}$, Wedel $\mathbf{H}$, et al. Intense metabolic control by means of insulin in patients with diabetes mellitus and acute myocardial infarction (DIGAMI 2): effects on mortality and morbidity. Eur Heart J 2005;26(7):650-661.

8. Action to Control Cardiovascular Risk in Diabetes Study G, Gerstein HC, Miller ME, et al. Effects of intensive glucose lowering in type 2 diabetes. $N$ Engl J Med 2008;358(24):2545-2559.
9. McCoy RG, Van Houten HK, Ziegenfuss JY, Shah ND, Wermers RA, Smith SA. Increased mortality of patients with diabetes reporting severe hypoglycemia. Diabetes Care 2012;35(9):1897-1901.

10. Zoungas S, Patel A, Chalmers J, et al. Severe hypoglycemia and risks of vascular events and death. N Engl J Med 2010;363(15):1410-1418.

11. Gamble JM, Simpson SH, Eurich DT, Majumdar SR, Johnson JA Insulin use and increased risk of mortality in type 2 diabetes: a cohort study. Diabetes Obes Metab 2010;12(1):47-53.

12. Currie CJ, Poole CD, Evans M, Peters JR, Morgan CL. Mortality and other important diabetes-related outcomes with insulin vs other antihyperglycemic therapies in type 2 diabetes. J Clin Endocrinol Metab 2013;98(2):668-677.

13. Roumie CL, Greevy RA, Grijalva CG, et al. Association between intensification of metformin treatment with insulin vs sulfonylureas and cardiovascular events and all-cause mortality among patients with diabetes. JAMA 2014;311(22):2288-2296.

14. Shorr RI, Ray WA, Daugherty JR, Griffin MR. Incidence and risk factors for serious hypoglycemia in older persons using insulin or sulfonylureas. Arch Intern Med 1997;157(15):1681-1686.

15. Garg R, Hurwitz S, Turchin A, Trivedi A. Hypoglycemia, with or without insulin therapy, is associated with increased mortality among hospitalized patients. Diabetes Care 2013;36(5):1107-1110.

16. Claesen M, Gillard P, De Smet F, Callens M, De Moor B, Mathieu C. Mortality in Individuals Treated With Glucose-Lowering Agents: A Large, Controlled Cohort Study. J Clin Endocrinol Metab 2016;101(2):461-469.

17. Lipska KJ, Ross JS, Wang Y, et al. National trends in US hospital admissions for hyperglycemia and hypoglycemia among Medicare beneficiaries, 1999 to 2011. JAMA Intern Med 2014;174(7):1116-1124.

18. Geller AI, Shehab N, Lovegrove MC, et al. National estimates of insulinrelated hypoglycemia and errors leading to emergency department visits and hospitalizations. JAMA Intern Med 2014;174(5):678-686.

19. American Diabetes Association. Standards of medical care in diabetes2016. Diabetes Care 2016;39 Suppl 1:S1-112.

20. Levy AR, O'Brien BJ, Sellors C, Grootendorst P, Willison D. Coding accuracy of administrative drug claims in the Ontario Drug Benefit database. Can J Clin Pharmacol= J Can Pharmacol Clin 2003;10(2):6771.

21. Deyo RA, Cherkin DC, Ciol MA. Adapting a clinical comorbidity index for use with ICD-9-CM administrative databases. J Clin Epidemiol 1992;45(6):613-619.

22. Schneeweiss S, Seeger JD, Maclure M, Wang PS, Avorn J, Glynn RJ Performance of comorbidity scores to control for confounding in epidemiologic studies using claims data. Am J Epidemiol 2001;154(9):854864.

23. van Walraven C, Dhalla IA, Bell C, et al. Derivation and validation of an index to predict early death or unplanned readmission after discharge from hospital to the community. CMAJ 2010;182(6):551-557.

24. van Walraven $\mathbf{C}$, Wong $\mathbf{J}$, Forster AJ. LACE+ index: extension of a validated index to predict early death or urgent readmission after hospital discharge using administrative data. Open Med 2012;6(3):e80-90.

25. Leong A, Berkowitz SA, Triant VA, et al. Hypoglycemia in Diabetes Mellitus as a Coronary Artery Disease Risk Factor in Patients at Elevated Vascular Risk. J Clin Endocrinol Metab 2016;101(2):659-668.

26. Juurlink DN, Mamdani MM, Lee DS, et al. Rates of hyperkalemia after publication of the Randomized Aldactone Evaluation Study. N Engl J Med 2004;351(6):543-551.

27. Healy SJ, Black D, Harris C, Lorenz A, Dungan KM. Inpatient diabetes education is associated with less frequent hospital readmission among patients with poor glycemic control. Diabetes Care 2013;36(10):29602967.

28. Wexler DJ, Beauharnais CC, Regan S, Nathan DM, Cagliero E, Larkin ME. Impact of inpatient diabetes management, education, and improved discharge transition on glycemic control 12 months after discharge. Diabetes Res Clin Pract 2012;98(2):249-256.

29. Braithwaite SS, Magee M, Sharretts JM, et al. The case for supporting inpatient glycemic control programs now: the evidence and beyond. J Hosp Med 2008;3(5 Suppl):6-16

30. Peyrot M, Rubin RR, Lauritzen T, et al. Resistance to insulin therapy among patients and providers: results of the cross-national Diabetes Attitudes, Wishes, and Needs (DAWN) study. Diabetes Care 2005;28(11):2673-2679.

31. Phillips CO, Wright SM, Kern DE, Singa RM, Shepperd S, Rubin HR. Comprehensive discharge planning with postdischarge support for older patients with congestive heart failure: a meta-analysis. JAMA 2004:291(11):1358-1367. 
32. Laverty AA, Elkin SL, Watt HC, et al. Impact of a COPD discharge care bundle on readmissions following admission with acute exacerbation: interrupted time series analysis. PLoS One 2015;10(2):e0116187.

33. Goncalves-Bradley DC, Lannin NA, Clemson LM, Cameron ID, Shepperd S. Discharge planning from hospital. Cochrane Database Syst Rev 2016(1):CD000313.

34. LaManna JB, Bushy A, Norris AE, Chase SK. Early and Intermediate Hospital-to-Home Transition Outcomes of Older Adults Diagnosed With Diabetes. The Diabetes Educator 2016;42(1):72-86.

35. Jack BW, Chetty VK, Anthony D, et al. A reengineered hospital discharge program to decrease rehospitalization: a randomized trial. Ann Intern Med 2009;150(3):178-187.

36. Coleman EA, Parry C, Chalmers S, Min SJ. The care transitions intervention: results of a randomized controlled trial. Arch Intern Med 2006;166(17):1822-1828.

37. Hansen LO, Young RS, Hinami K, Leung A, Williams MV. Interventions to reduce 30-day rehospitalization: a systematic review. Ann Intern Med 2011;155(8):520-528.

38. Seggelke SA, Hawkins RM, Gibbs J, Rasouli N, Wang C, Draznin B Transitional care clinic for uninsured and medicaid-covered patients with diabetes mellitus discharged from the hospital: a pilot quality improvement study. Hosp Pract 2014;42(1):46-51.
39. Dungan $\mathbf{K}$, Lyons $\mathbf{S}$, Manu $\mathbf{K}$, et al. An individualized inpatient diabetes education and hospital transition program for poorly controlled hospitalized patients with diabetes. Endocr Pract : Off J Am Coll Endocrinol Am Assoc Clin Endocrinol 2014;20(12):1265-1273.

40. Rubin DJ, Donnell-Jackson K, Jhingan R, Golden SH, Paranjape A. Early readmission among patients with diabetes: a qualitative assessment of contributing factors. J Diabetes Complicat 2014;28(6):869-873.

41. Lipska KJ, Ross JS, Miao Y, Shah ND, Lee SJ, Steinman MA. Potential overtreatment of diabetes mellitus in older adults with tight glycemic control. JAMA Intern Med 2015;175(3):356-362.

42. McCoy RG, Lipska KJ, Yao X, Ross JS, Montori VM, Shah ND. Intensive Treatment and Severe Hypoglycemia Among Adults With Type 2 Diabetes. JAMA Intern Med 2016;176(7):969-978.

43. Rodriguez-Gutierrez R, Lipska KJ, McCoy RG. Intensive Glycemic Control in Type 2 Diabetes Mellitus-A Balancing Act of Latent Benefit and Avoidable Harm: A Teachable Moment. JAMA Intern Med 2016;176(3):300-301.

44. Jackevicius CA, Mamdani M, Tu JV. Adherence with statin therapy in elderly patients with and without acute coronary syndromes. JAMA 2002;288(4):462-467. 